Le diagnostic institutionnel et la méthodologie de développement institutionnel : l'expérience du projet d'appui aux ONGs/USAID au Sénégal

Awa Paye Guèye et Massamba Dieng

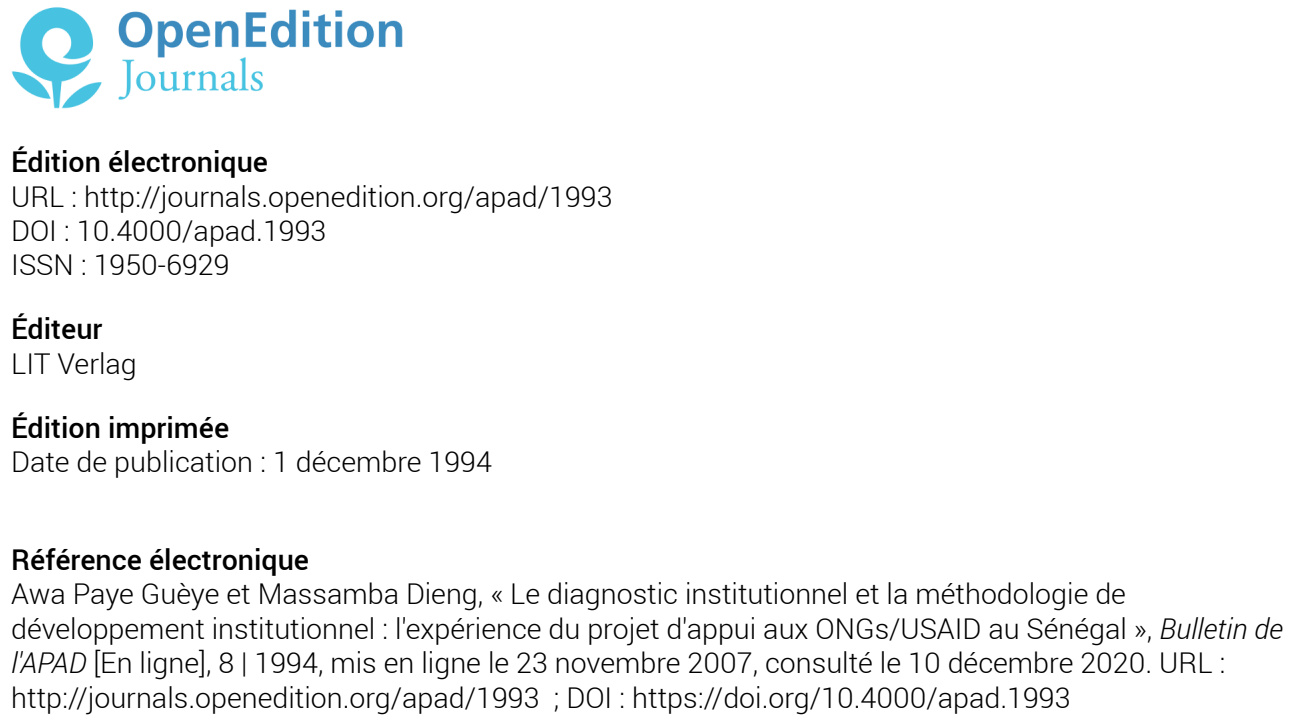

Ce document a été généré automatiquement le 10 décembre 2020.

Bulletin de l'APAD 


\title{
Le diagnostic institutionnel et la méthodologie de développement institutionnel : l'expérience du projet d'appui aux ONGs/USAID au Sénégal
}

\author{
Awa Paye Guèye et Massamba Dieng
}

Le contexte

1 Face aux déséquilibres macro-économiques et macro-financiers persistants et pour tenter d'enrayer le processus de dégradation des finances publiques et de relancer la croissance économique d'une façon durable, le Gouvernement du Sénégal a entrepris, depuis la fin des années 1970, une série de réformes économiques.

2 Par le Plan de Redressement Economique et Financier, lancé en novembre 1979, l'Etat a cherché à stabiliser les équilibres macro-financiers par l'assainissement des finances publiques.

3 Le faible impact de ce Plan sur la stagnation des secteurs productifs, notamment du secteur primaire, amena le Gouvernement à adopter un Plan d'Ajustement Structurel à Moyen et Long Termes, couvrant la période 1985 - 1992, avec comme axe principal une profonde réorganisation des secteurs productifs.

4 La mise en œuvre du Programme d'Ajustement Structurel, qui s'est concrétisée à travers la Nouvelle Politique Agricole et la Nouvelle Politique Industrielle, a comporté un certain nombre de mesures dont:

- une réduction sensible de l'intervention de l'Etat dans les sphères de la production et du commerce ;

- une plus grande libéralisation des marchés ;

- la réduction des subventions aux producteurs ;

- l'incitation du secteur privé à assumer plus de fonctions et de responsabilités dans la production. 
5 Au niveau du secteur rural, le désengagement de l'Etat qui s'est opéré à travers le dépérissement de ses organismes d'encadrement a été marqué par un vide pour ce qui concerne la fourniture d'intrants, de divers services et de l'assistance technique aux producteurs. Les Organisations Non Gouvernementales ONGs et les organisations villageoises qui interviennent à la base ont tout de suite tenté de combler le vide créé par le retrait de l'Etat, en élargissant au maximum leurs domaines d'intervention. Elles ont également cherché à mieux mobiliser les ressources locales.

6 Ce nouvel environnement a alors permis un essor des ONGs et des organisations villageoises qui sont devenues, au cours des années 80 , des cadres de développement reconnus comme des pièces essentielles pour susciter et promouvoir la participation du plus grand nombre, notamment les couches les plus démunies, au développement économique et social.

7 La reconnaissance du phénomène des ONGs et des organisations villageoises, aussi bien par les pouvoirs publics que par les bailleurs de fonds, a eu comme effet un changement d'approche de ceux-ci dans leurs modes d'intervention et de financement du développement rural sénégalais.

8 En effet, les ONGs et les organisations villageoises, considérées jusqu'à cette mutation comme des concurrentes des structures publiques d'encadrement rural, sont devenues de plus en plus des partenaires dont l'action s'inscrit désormais dans un cadre légal, avec notamment la loi sur les Groupements d'Intérêt Economiques (GIE) et le décret fixant les modalités d'intervention des ONGs.

Dans la mise en œuvre de cette mutation, les agences de financement bilatérales et multilatérales ont initié, au cours des dernières années, des programmes d'appui aux ONGs et aux organisations villageoises, afin de conférer progressivement à ces dernières, un rôle d'intermédiaire pour toucher avec plus d'impact les communautés de base.

10 A partir de ce moment, l'environnement du développement rural est-il devenu plus incitatif pour la promotion et le développement des ONGs, des organisations villageoises et de leurs fédérations.

11 Cela s'est traduit par un accroissement très rapide du nombre d'ONGs qui interviennent au Sénégal. D'une vingtaine dans les années 70, le nombre des ONGs est passé aujourd'hui à plus de 250 dont 180 au moins sont reconnues et enregistrées auprès du Ministère de la Femme, de l'Enfant et de la Famille (MFEF), ministère de tutelle.

On note que par rapport à certains pays africains, le phénomène des ONGs au Sénégal apparaît comme relativement jeune malgré son ambition de se muer en un mouvement à la base fort et crédible par des regroupements qui se constituent pour accompagner la croissance et la maturation dudit mouvement.

13 C'est ainsi que, certaines fédérations d'ONGs telles que la Fédération des Organisations Non-Gouvernementales du Sénégal (FONGS) et le Conseil des Organisations Non-Gouvernementales d'Appui au Développement (CONGAD) sont parvenues à être de plus en plus consultées et associées au moment de la définition des politiques de développement et de leur suivi.

Mais, actuellement force est de reconnaître qu'en dépit de tous les efforts déployés, cette maturation souhaitée des fédérations est très lente particulièrement par manque 
de vision, de méthodes et de stratégies adaptées pour soutenir leurs organisations membres naissantes. niveau de vie. institutionnel. octroyé.

Cest dans ce cadre général que s'inscrit la création du Projet d'Appui aux ONGs financé par l'USAID pour un montant de 21 millions de dollars US et une durée de 8 ans.

L'objectif principal que les concepteurs de ce Projet se sont fixé était de créer le meilleur cadre susceptible de développer la capacité des communautés de base et des populations les plus démunies, à mobiliser leurs propres ressources en vue de planifier et de mener elles-mêmes des activités de développement viables qui élèvent leur

L'intervention de structures intermédiaires telles que les ONGs pour aider à réaliser cette finalité dans le contexte décrit ci-dessus a naturellement posé de manière cruciale la question du choix de méthodologies appropriées et adaptées tant pour la sélection des avant-projets que pour la promotion d'organes d'exécution stables sur le plan

Pour cela, l'approche que la stratégie du projet a choisie est celle du développement institutionnel des ONGs et des groupements villageois comme le moyen privilégié pour participer avec efficacité au développement à la base durable.

La présentation du projet d'appui aux ongsLe but et les objectifs du Projet

Le But du programme dans lequel s'inscrit le Projet d'Appui aux ONGs est d'améliorer le niveau de vie des populations sénégalaises les plus démunies.

L'objectif spécifique qui a été fixé au Projet dans ce programme d'intervention de l'USAID au Sénégal le "Country Program Strategie Plan (CPSP)", est le suivant :

améliorer les compétences et renforcer la capacité des ONGs locales, des associations d'ONGs et des groupements communautaires, à planifier, concevoir et réaliser des activités de développement viables avec la participation des populations, avec ou sans la collaboration d'ONGs américaines.

La démarche qui a été suggérée dans le document de conception de projet pour atteindre cet objectif a tiré toutes les leçons des expériences que l'USAID a eues dans un passé plus ou moins récent avec les ONGs et les Projets de développement.

Les principales faiblesses qui ont été constatées dans ces expériences au Sénégal et ailleurs sont relatives au faible impact que les programmes de crédits ou de subventions ont eu sur les activités et sur les organisations.

Ce double échec quasi-général est particulièrement visible pour ce qui concerne :

- d'une part, à la pérennité des activités de développement ;

- d'autre part, à la survie des organisations bénéficiaires à la fin de la subvention ou du crédit

Ces constats ont été confirmés par les craintes et attentes qui ont été exprimées par la communauté des ONGs du Sénégal au moment de l'élaboration du document de Projet et pendant les études de base sur les besoins en formation, les réseaux d'ONGs et le partenariat qui ont été réalisées auprès de cette communauté.

C'est certainement pour répondre à cette double problématique de l'impact des activités financées sur les conditions de vie des populations et des organisations bénéficiaires, que deux volets ont été choisis comme axes d'intervention de l'USU :

- d'une part, la subvention d'activités de développement ; 
- d'autre part, le financement du développement institutionnel des ONGs par la formation et

l'assistance technique.

\section{rendue par quelques concepts-clés liés à des activités productives et sociales viables} réalisées par des organisations locales de plus en plus autonomes. A savoir :

- une implication soutenue des bénéficiaires depuis la phase de conception du projet soumis;

- une concentration des efforts sur la capacité de gestion des ONGs et des groupements pour

l'exécution du projet soumis;

- une promotion de la collaboration entre les ONGs et tous les acteurs à la base y compris les services administratifs décentralisés ;

- la transparence et l'égalité dans le traitement des demandes de subventions à l'aide de méthodologies appropriées ;

- l'encouragement de systèmes efficaces de financement d'activités productives au niveau local par un système de Fonds de Roulement sous forme de crédit Les méthodologies de l'USU pour se conformer aux principes de base et à la stratégie ont privilégié l'approche participative tant au niveau du processus de sélection et de gestion des subventions qu'au niveau du programme de développement institutionnel des organisations.

les études de base et les leçons tirées

Au cours de la première année du projet, trois études de base ont été menées auprès de la communauté des ONGs pour établir une situation de référence et faire le point sur 
certaines recommandations qui ont été formulées par les évaluations des expériences antérieures déjà citées. Il s'agit des études suivantes :

- l'étude des besoins des ONGs en formation et en appui institutionnel ;

- l'étude du Partenariat en milieu ONGs au Sénégal ;

- l'étude du développement des réseaux en milieu ONGs au Sénégal.

Partie intégrante de la stratégie préliminaire de développement institutionnel qui couvrent les 18 premiers mois du projet, ces trois études ont permis de déceler les contraintes spécifiques auxquelles les ONGs, les réseaux et les instituts de formation qui interviennent au Sénégal font face.

L'un des principaux constats de ces études est l'existence d'une certaine stratification des ONGs du Sénégal sur la base de leur niveau de Développement :

- ONGs en phase de formation ;

- ONGs en phase de développement ;

- ONGs en phase d'autogestion.

Une telle classification qui rend compte, pour les éléments d'un même groupe, sur leurs caractéristiques, capacités et besoins spécifiques, a un intérêt certain pour la conception d'un programme de renforcement qui se veut adapté et durable.

L'approche participative dans le processus de développement institutionnel des organisations à la base

Pour favoriser l'émergence d'ONGs efficaces et viables, les activités initiées par l'USU dans ce sens visent à développer les capacités des ONGs, afin de leur permettre d'acquérir les compétences et des approches adaptées pour aider les populations à trouver des solutions durables aux besoins de développement.

Il faut noter qu'au départ deux contraintes de tailles ne rendaient pas cet objectif de développement institutionnel des ONGs, facile d'accès :

- d'une part, le Projet dispose de ressources financières limitées face à une demande très forte des ONGs, réseaux, associations et fédérations ;

- d'autre part, le Projet a l'ambition de développer, en même temps que la subvention, un programme d'appui institutionnel comme moyen privilégié pour la pérennisation des activités et des organisations.

Le processus de sélection des demandes de subventions a constitué le cadre dans lequel l'USU a créé, avec les ONGs présélectionnées, un courant de communication qui génère l'information utile pour établir une situation de référence des dites organisations. Cette situation de référence servira de base à la mise en œuvre du programme d'appui aux organisations bénéficiaires.

C'est ainsi que dès la phase de présélection des documents de projets, un poids relativement important a été donné aux critères d'appréciation de la faisabilité sociale du projet soumis et de l'approche de l'organisation à côté des critères généraux d'éligibilité. Ces derniers sont relatifs aux secteurs d'activité, à l'approbation locale, à la rentabilité financière et à la faisabilité technique.

Après cette présélection des dossiers, la phase suivante du processus consiste en des études de diagnostic participatif auprès des groupements bénéficiaires et auprès des organisations dont les propositions ont été retenues.

L'étude d'impact 


\section{Développement Institutionnel de l'USU qui fait l'objet de la présente communication, dans ses aspects innovateurs pour un développement à la base durable et centré sur le} bénéficiaire.

Le diagnostic institutionnel participatif (DIP)

51 Il faut d'abord préciser que cette méthodologie est le produit d'une recherche de l'équipe du Projet dirigée par Mary Ann Zimmerman C.O.P et appuyée par Maty NDIAYE, une consultante de nationalité sénégalaise spécialisée dans le développement des ressources humaines. 
publication de fascicules sur cette expérience de l'USU qui seront mis à la disposition de la communauté des ONGs et des autres intéressés.

\section{l'organisation}

• il va à l'essentiel ;

- il donne une photographie instantanée ;

- son processus de cinq phases est brusque (la mise au point, la découverte, l'exploration, le diagnostic et les perspectives) avec trois séances collectives (le focus, le scanner et la restitution). 

ont indiqué de manière précise les informations qui sont recherchées, mais le DIP doit resté flexible.

- il s'adapte aux interlocuteurs, aux ONGs diverses ;

- il utilise avec souplesse les outils tel que le guide d'entretien;

- il fait appel à l'intuition pour évoluer sur le terrain.

\section{Le DIP est pertinent}

- il doit avoir un impact certain ;

- il est un outil de décision ;

- il est un outil de gestion (partenariat) ;

- il est un appui institutionnel en soi-même

- il doit changer la perspective de l'ONG sur le management, l'équipe, l'environnement et le projet soumis ;

- il renforce institutionnellement car il est formatif.

\section{Le DIP est donc très exigeant}

- pour le client, l'ONG (disponibilité, implication, sincérité, esprit positif et ouvert au changement);

- pour l'USU ou son consultant (sens de la pédagogie, engagement, esprit critique, capacité d'écouter, de négocier, de persuader et de se remettre en cause, maîtrise du domaine). Les principaux outils du DIP telles que la définition claire et partagée des objectifs de la recherche, du profil de l'information recherchée, du choix des outils etc...

Les principaux outils que le DIP exploite sont les suivants :

- le processus, favorisant une approche participative ;

- la démarche, privilégiant la transparence de la méthode et des résultats ;

- le guide d'entretien, check-list permettant d'explorer les différents aspects de

l'organisation;

- une technique d'écoute, mettant l'accent sur la reformulation et la compréhension;

- des séances collectives, n'excédant pas une douzaine de participants ;

- une méthode d'animation rigoureuse, alternant une procédure d'expression individuelle, des échanges en petits groupes, des séances plénières, des entretiens ;

- la technique du feed-back particulièrement aux séances scanner ou miroir, et aux séances de restitution ; 
- l'écrit, notamment les comptes-rendus préparant le diagnostic et les restitutions ;

- les évaluations, à mi-parcours et à la fin du séjour ;

- la restitution comme moyen de faire valider les constats et conclusions

- l'étude Certification Financière, donnant un éclairage sur les opérations et procédures

administratives et financières.

Les attitudes à privilégier au cours du DIP pour le consultant qui se positionne comme agent de changement donc supposé détenir l'expertise exigée pour exploiter au mieux le vécu de l'enquêté. L'enquêté qui devra également valider au fur et à mesure tous les constats de la recherche. attitudes suivantes qui sont attendues de ce dernier au cours du DIP :

- écouter, chercher constamment à comprendre ce que dit l'autre, s'efforcer à s'introduire dans le problème tel qu'il est vécu par l'autre ;

- être flexible, ne pas hésiter à changer une modalité de travail, un horaire, une date planifiée si le contexte le demande ;

- provoquer des situations ou les interlocuteurs sont conduits alternativement à prendre du recul et à s'impliquer ;

- favoriser la transparence, notamment en expliquant la procédure et en faisant un feed-back au fur et à mesure des problèmes évoqués car cela crée la confiance et la sécurité ;

- avoir des attitudes positives qui favorisent des comportements propices au changement, l'évolution des opinions ;

- faire preuve de discrétion ;

- ne pas avoir d'à priori, être ouvert.

Le guide d'entretien

Le guide d'entretien ou check-list pennet un inventaire exhaustif de sept principales dimensions suivantes de l'organisation :

- identité de l'organisation ;

- la mission de l'organisation ;

- l'environnement interne et externe ;

- le management ;

- les opérations internes ;

- les moyens/ressources ;

- les services fournis et résultats atteints. collecter qui fait que le guide est également une liste de contrôle. premiers cycles de subventions. Le consultant qui l'utilise est tour à tour facilitateur (séances collectives) et interviewer. La combinaison de plusieurs sources pour une même information est un moyen de rapprochement qui est utilisé systématiquement pour limiter au mieux les biais possibles dans une recherche aussi intensive et participative. C'est ainsi que la CF éclaire certaines dimensions telles que les opérations, les moyens et l'environnement interne.

Conclusion 
Il est évident que le DIP tel que décrit ci-dessus n'est mis en œuvre que pour les ONGs du Sénégal qui sont dans le processus de sélection des subventions, il n'en touchera donc qu'un nombre relativement réduit.

En effet, le nombre d'ONGs à qui le Projet prévoit de donner une subvention ne dépasse pas la cinquantaine or le programme d'appui institutionnel est conçu pour avoir un impact sur toute la communauté des ONGs.

La stratégie de développement institutionnel de l'USU a imaginé plusieurs axes d'intervention adaptés d'une part à la diversité de niveaux de développement, et d'autre part au bénéfice ou non de la subvention.

3 Le programme distingue alors les ONGs bénéficiaires du reste de la communauté qui est entendu ici comme toutes les autres ONGs non bénéficiaires de subvention mais qui ont été en contact avec les activités du Projet notamment à travers les ateliers de formation offerts.

4 Sans insister outre mesure sur les différents contenus et définitions qui sont données aux concepts relatifs au développement institutionnel, on peut brièvement présenter, dans cette dernière partie de la communication, la stratégie de développement institutionnel telle qu'elle a été mise en œuvre par l'USU dans le contexte des ONGs au Sénégal.

Les résultats du dip et le développement institutionnel des ONGsLe programme de développement institutionnel et les ONGs bénéficiaires

Les études d'impact, de DIP et de CF effectuées auprès de ces organisations constituent les principales sources d'identification du type d'appui spécifique qu'il convient de leur fournir ainsi que les meilleures modalités pour le faire. La réalisation du projet subventionné constitue également un moyen de mesure de l'impact de cet appui sur l'organisation et les populations bénéficiaires.

86 La stratégie de l'USU a distingué deux principales branches du Développement Institutionnel (DI), en fonction de la cible :

- l'Appui Institutionnel (AI), qui concerne les ONGs en création et les ONGs informelles caractérisées par une grande faiblesse organisationnelle. Il s'adresse également aux ONGs en phase de développement ou ayant acquis un certain niveau d'expérience dans la gestion d'une activité de développement, mais insuffisamment développées au plan organisationnel pour pouvoir fonctionner de manière durable ;

- le Renforcement Institutionnel (RI) concerne les ONGs qui ont une grande expérience en planification et gestion de projets à volets multiples et qui ont déjà la pratique de méthodes de gestion administrative et financière.

87 L'Appui Institutionnel est une assistance qui est censée comprendre les activités et toutes les ressources mobilisées en vue de développer et de renforcer des organisations existantes qui, bien que justifiant d'un certain potentiel, disposent de ressources humaines, matérielles et financières limitées pour assurer leur mission.

Cette assistance peut se faire sous forme d'ateliers de formation (gestion des projets, gestion des organisations, gestion de la croissance, etc...) d'assistance technique (mise en place de systèmes de gestion) et d'assistance financière pour :

- aider à la création d'organisations formelles ;

- aider à l'élaboration des méthodes minimales pour assurer une gestion administrative et financière appropriée de l'organisation et de ses activités ;

- prendre en charge et valoriser les ressources humaines indispensables à l'organisation. 
Les activités de renforcement institutionnel sont sensées contribuer à l'augmentation de l'impact des ONGs ayant atteint un certain degré de maturité ou étant parvenues à ce qu'on pourrait appeler la phase d'auto-développement.

En effet, certaines de ces organisations seront encouragées par le RI à évoluer du statut de partenaire à celui de ressource pour l'appui aux membres de la communauté des ONGs dans des domaines de compétences distinctives. En général, elles disposent déjà de structures et de personnel appropriés pour l'exécution de projets, mais elles souhaitent se spécialiser dans un domaine technique ou explorer de nouveaux secteurs.

9 Ces ONGs sont dites en phase d'autogestion et ont parfois un objectif d'autonomisation des communautés qu'elles ont appuyées pendant longtemps, en procédant à un transfert de compétence et de responsabilité.

La forme que prend cette assistance de renforcement est tributaire des résultats du DIP qui est suivi de la formation du staff de l'ONG à ladite méthodologie pour l'élaboration puis l'exécution d'un plan de développement institutionnel.

Le programme de développement institutionnel et la communauté des ONGs

3 Pour atteindre l'impact recherché sur la communauté des ONGs, en plus du DIP, le programme de développement institutionnel du Projet a du recourir à des axes complémentaires d'intervention.

Dans ce cadre on pourrait citer :

- le fait de restituer les rapports des études de base (besoins, réseaux et partenariat) pour qu'ils soient validés par les ONGs qui y avaient participé ;

- la mise en place d'un Comité Consultatif pour recevoir un éclairage d'experts indépendants sur tous les programmes et projets entrepris dans ce domaine ;

- la réalisation d'un programme de formation spécifique à la communauté des ONGs dans lequel intervient les réseaux, consortia et institutions de formation ;

- l'élargissement de la base des consultants qui maîtrisent les méthodologies de conception et d'exécution des projets, par la formation des formateurs et le système de stagiaires-formateurs ;

- le financement des projets de partenariat entre ONGs du Nord et ONGs locales qui a fait l'objet d'une attention particulière dans la conception du Projet.

C'est dans le même ordre d'idée qu'il faut placer la sélection des deux éléments discriminatoires qui guident l'intervention en matière de développement institutionnel :

- la stratification des ONGs en fonction de leur niveau de développement ;

- le bénéfice ou non de la subvention.

Dans la compréhension du programme d'appui, la communauté des ONGs n'est pas constituée par toutes les ONGs qui interviennent au Sénégal, mais par celles qui, sans être bénéficiaires de subvention sont néanmoins en contact avec le Projet à travers les activités de formation et de présélection. Elles sont environ une soixantaine.

Une bonne partie de cet appui à la communauté des ONGs passe également par d'autres organisations telles que les réseaux et consortia ou les instituts de formation.

Cette approche a exigé du Projet le choix de critères de sélection centrés sur le niveau et la pertinence de l'appui institutionnel que ces ressources sont en mesure d'apporter en retour à la communauté des ONG tant à l'heure actuelle que dans l'avenir. 
99 A travers ces organisations ressources et le processus qui est développé, sont visées toutes les potentialités qui ont été identifiées lors des études de base afin d'assurer la pérennité des activités et des organisations pour un développement à la base durable. 